\title{
Quasi-constricted linear operators on Banach spaces
}

\author{
by \\ Eduard Yu. EMEL'Yanov (Novosibirsk and Tübingen) and \\ Manfred P. H. WolfF (Tübingen)
}

\begin{abstract}
Let $X$ be a Banach space over $\mathbb{C}$. The bounded linear operator $T$ on $X$ is called quasi-constricted if the subspace $X_{0}:=\left\{x \in X: \lim _{n \rightarrow \infty}\left\|T^{n} x\right\|=0\right\}$ is closed and has finite codimension. We show that a power bounded linear operator $T \in L(X)$ is quasi-constricted iff it has an attractor $A$ with Hausdorff measure of noncompactness $\chi_{\|\cdot\|_{1}}(A)<1$ for some equivalent norm $\|\cdot\|_{1}$ on $X$. Moreover, we characterize the essential spectral radius of an arbitrary bounded operator $T$ by quasi-constrictedness of scalar multiples of $T$. Finally, we prove that every quasi-constricted operator $T$ such that $\bar{\lambda} T$ is mean ergodic for all $\lambda$ in the peripheral spectrum $\sigma_{\pi}(T)$ of $T$ is constricted and power bounded, and hence has a compact attractor.
\end{abstract}

1. Introduction. Let $(X,\|\cdot\|)$ be a Banach space over the field $\mathbb{C}$ of complex numbers. We denote the space of all bounded linear operators on $X$ by $L(X)$. Let $T \in L(X)$. A subset $A \subseteq X$ is called an attractor for $T$ if $\lim _{n \rightarrow \infty} \operatorname{dist}\left(T^{n} x, A\right)=0$ for each $x \in B_{X}$, where $B_{X}$ is the closed unit ball of $X$ and $\operatorname{dist}(y, A)$ denotes the distance $\inf \{\|y-z\|: z \in A\}$ from $y$ to $A$. We will denote the set of all attractors for $T$ by $\operatorname{Attr}_{\|\cdot\|}(T)$. It was established by many authors (see, for example, [LLY], [Ba], [Si]) that a power bounded operator $T \in L(X)$ has a compact attractor iff there exists a decomposition $X:=X_{0} \oplus X_{r}$ of the Banach space $X$ into $T$-invariant subspaces $X_{0}$ and $X_{r}$ such that $X_{0}=\left\{x \in X: \lim _{n \rightarrow \infty}\left\|T^{n} x\right\|=0\right\}$ and $\operatorname{dim}\left(X_{r}\right)<\infty$. Operators of this type are called constricted.

Here we will study bounded linear operators $T$ on $X$ which satisfy the weaker condition that the subspace $X_{0}:=\left\{x \in X: \lim _{n \rightarrow \infty}\left\|T^{n} x\right\|=0\right\}$ of $X$ is closed and has finite codimension. We call these operators quasiconstricted. Our first main result (Theorem 1) characterizes these operators in the following way: a power bounded $T \in L(X)$ is quasi-constricted iff there exists an attractor for $T$ which has Hausdorff measure of noncompactness $\chi_{\|\cdot\|_{1}}(A)<1$ for some equivalent norm $\|\cdot\|_{1}$ on $X$. Moreover, we show that for each $\varepsilon>0$ there exists an $\varepsilon$ - $T$-invariant subspace complementary

2000 Mathematics Subject Classification: Primary 47A65; Secondary 47A35, 47A10. 
to $X_{0}$. Here a subspace $Y$ is called $\varepsilon$-T-invariant if for every $y \in Y$ we have $\operatorname{dist}(T y, Y) \leq \varepsilon\|T y\|$.

We study the spectrum of a quasi-constricted operator and conclude that for such an operator $T, \lim _{n \rightarrow \infty} \operatorname{dist}\left(T^{n} x, X_{0}\right)=0$ always implies $x \in X_{0}$. Moreover, we characterize the essential spectral radius $r_{\text {ess }}(T)$ of an arbitrary bounded operator $T$ by the quasi-constrictedness of scalar multiples of $T$.

Let $T$ be quasi-constricted. Then $T$ need not be constricted as easy examples show. However, in Theorem 7 we show that if the operators $\bar{\lambda} T$ are mean ergodic for all $\lambda$ in the peripheral spectrum $\sigma_{\pi}(T)$ of $T$ then $T$ must be power bounded and constricted.

\section{Quasi-constricted operators and their attractors}

Theorem 1. Let $X$ be a Banach space and let $T \in L(X)$ be a power bounded operator. Then the following conditions are equivalent:

(i) $T$ is quasi-constricted.

(ii) For every $\varepsilon>0$ there exists a finite-dimensional $\varepsilon$-T-invariant subspace $Y$ with $X=X_{0} \oplus Y$.

(iii) For every finite-dimensional subspace $Y$ with $X=X_{0} \oplus Y$ and for every $\varepsilon>0$ there exists $n \in \mathbb{N}$ such that $T^{n}(Y)$ is $\varepsilon$-T-invariant and $X=X_{0} \oplus T^{n}(Y)$.

(iv) For every $\varepsilon>0$ there exists an equivalent norm $\|\cdot\|_{\varepsilon}$ on $X$ and $A_{\varepsilon} \in \operatorname{Attr}_{\|\cdot\|_{\varepsilon}}(T)$ such that its Hausdorff measure $\chi_{\|\cdot\|_{\varepsilon}}\left(A_{\varepsilon}\right) \leq \varepsilon$.

(v) There exists an equivalent norm $\|\cdot\|_{1}$ on $X$ and $A \in \operatorname{Attr}_{\left\|_{1}\right\|_{1}}(T)$ such that $\chi_{\|\cdot\|_{1}}(A)<1$.

Proof. (i) $\Rightarrow$ (iv). Take some $\varepsilon>0$ and let $P_{0}$ be a projection of $X$ onto $X_{0}$. Let $P:=I-P_{0}$. Define $M:=\left\|P_{0}\right\| \sup _{n \in \mathbb{N}}\left\|T^{n}\right\|$ and consider the equivalent norm $\|\cdot\|_{\varepsilon}$ on $X$ :

$$
\|y\|_{\varepsilon}:=\|P y\|+\varepsilon M^{-1}\left\|P_{0} y\right\| \quad(y \in X) .
$$

Take $y \in X,\|y\|_{\varepsilon} \leq 1$. Then $P T^{n} P y \in P T^{n}\left(B_{X}\right) \subseteq M P\left(B_{X}\right)$ for all $n \in \mathbb{N}$. Consequently,

$$
\begin{aligned}
\varlimsup_{n \rightarrow \infty} \operatorname{dist}_{\|\cdot\|_{\varepsilon}}\left(T^{n} y, M P\left(B_{X}\right)\right) & \leq \varlimsup_{n \rightarrow \infty}\left\|T^{n} y-P T^{n} P y\right\|_{\varepsilon} \\
& =\varlimsup_{n \rightarrow \infty}\left\|T^{n} P y-P T^{n} P y\right\|_{\varepsilon} \\
& =\varlimsup_{n \rightarrow \infty}\left\|P_{0} T^{n} P y\right\|_{\varepsilon}=\varepsilon M^{-1} \varlimsup_{n \rightarrow \infty}\left\|P_{0} T^{n} P y\right\| \\
& \leq \varepsilon\|P y\| \leq \varepsilon\|y\|_{\varepsilon} \leq \varepsilon .
\end{aligned}
$$

The set $M P\left(B_{X}\right)$ is compact, since $\operatorname{dim}(P(X))<\infty$. So we deduce that $A_{\varepsilon}:=M P\left(B_{X}\right)+\left\{y \in X:\|y\|_{\varepsilon} \leq \varepsilon\right\}$ is an attractor for $T$ that satisfies $\chi_{\|\cdot\|_{\varepsilon}}\left(A_{\varepsilon}\right) \leq \varepsilon$. 
(iv) $\Rightarrow(\mathrm{v})$. Obvious.

$(\mathrm{v}) \Rightarrow(\mathrm{i})$. Without loss of generality we assume that $\|\cdot\|_{1}$ is the initial norm $\|\cdot\|$ on $X$. Take a free ultrafilter $\mathcal{U}$ on $\mathbb{N}$ and consider the bounded ultrapower $X_{\mathcal{U}}:=\ell^{\infty}(X) / c_{\mathcal{U}}(X)$, where $\ell^{\infty}(X):=\left\{\left(x_{n}\right) \in X^{\mathbb{N}}: \sup \left\{\left\|x_{n}\right\|\right.\right.$ : $n \in \mathbb{N}\}<\infty\}$ and $c_{\mathcal{U}}(X):=\left\{\left(x_{n}\right) \in X^{\mathbb{N}}: \lim _{\mathcal{U}}\left\|x_{n}\right\|=0\right\}$. Then $X_{\mathcal{U}}$ is a Banach space with respect to the norm $\left\|\widehat{\left(x_{n}\right)}\right\|:=\lim _{\mathcal{U}}\left\|x_{n}\right\|$. We will identify $X$ with the subspace in $X_{\mathcal{U}}$ of all equivalence classes of constant sequences. Consider the linear operator $\mathcal{T}: X \rightarrow X_{\mathcal{U}}$ defined by $\mathcal{T} x:=\left(\widehat{T^{n} x}\right)$ for all $x \in X$ and let $Z:=\mathcal{T}(X)$. Take a real $\delta>0$ such that $(1+\delta) \alpha<1$ where $\chi(A)<\alpha<1$ and let the set $\left\{a_{i}\right\}_{i=1}^{p} \subseteq X$ satisfy $A \subseteq \bigcup_{i=1}^{p} B\left(a_{i}, \alpha\right)$.

Take an arbitrary $x=\mathcal{T} y \in B_{Z}$. Then $\left\{n:\left\|T^{n} y\right\|<1+\delta / 2\right\} \in \mathcal{U}$, and consequently, there exists some $m \in \mathbb{N}$ that satisfies $\left\|T^{m} y\right\|<1+\delta / 2$. Since

$$
\lim _{n \rightarrow \infty} \operatorname{dist}\left(T^{n} T^{m} y, \bigcup_{i=1}^{p} B\left(b_{i},(1+\delta / 2) \alpha\right)=0\right.
$$

where $b_{i}:=(1+\delta / 2) a_{i}$, there exists $i_{x} \in \overline{1, p}$ such that

$$
\left\{k \in \mathbb{N}:\left\|T^{k} y-b_{i_{x}}\right\|<(1+\delta) \alpha\right\} \in \mathcal{U} .
$$

So, we have $\left\|x-b_{i_{x}}\right\| \leq(1+\delta) \alpha$ in $X_{\mathcal{U}}$. Consequently,

$$
\left.B_{Z} \subseteq \bigcup_{i=1}^{p} B\left(b_{i},(1+\delta) \alpha\right)\right)
$$

Now [LT, Lemma 2.c.8] implies that $\operatorname{dim} Z<\infty$, since $(1+\delta) \alpha<1$.

Since the operator $T$ is power bounded, we may assume without loss of generality that $\|T\| \leq 1$. Therefore for each $x \in X$ the (nonnegative) sequence $\left(\left\|T^{n} x\right\|\right)_{n=1}^{\infty}$ is decreasing, and hence converges. Assume that $\lim _{\mathcal{U}}\left\|T^{n} x\right\|=0$. Since the limit of a sequence according to an ultrafilter is a limit point of the sequence and since the sequence $\left(\left\|T^{n} x\right\|\right)_{n=1}^{\infty}$ converges, it follows that $\lim _{n \rightarrow \infty}\left\|T^{n} x\right\|=0$, which proves that $\operatorname{ker} \mathcal{T} \subseteq X_{0}:=$ $\left\{x \in X: \lim _{n \rightarrow \infty}\left\|T^{n} x\right\|=0\right\}$. The inverse inclusion is trivial. The equality $X_{0}=\operatorname{ker} \mathcal{T}$ implies that $X_{0}$ is closed and that $\operatorname{codim}\left(X_{0}\right)=\operatorname{dim} Z<\infty$. Consequently, $T$ is quasi-constricted.

(iii) $\Rightarrow($ ii $) \Rightarrow($ i). Obvious.

(i) $\Rightarrow$ (iii). Let $X_{\mathcal{U}}$ and $\mathcal{T}: X \rightarrow X_{\mathcal{U}}$ be defined as in part (v) $\Rightarrow$ (i) of the proof. Let $Y$ be an arbitrary algebraic complement of $X_{0}$ in $X$. Obviously, $T^{n}(Y) \oplus X_{0}=X$ for all $n \in \mathbb{N}$.

Now let $\varepsilon>0$. If the assertion does not hold then for every $n \in \mathbb{N}$ there exists a normalized $y_{n} \in Y$ such that $\operatorname{dist}\left(T\left(T^{n} y_{n}\right), T^{n}(Y)\right) \geq \varepsilon\left\|T^{n+1} y_{n}\right\|$. 
The unit sphere of $Y$ is compact, so $\lim _{\mathcal{U}} y_{n}=y$ exists. But then

$$
\begin{aligned}
\lim _{\mathcal{U}} \operatorname{dist}\left(T^{n+1} y, T^{n}(Y)\right) & =\lim _{\mathcal{U}} \operatorname{dist}\left(T^{n+1} y_{n}, T^{n}(Y)\right) \\
& \geq \varepsilon \lim _{\mathcal{U}}\left\|T^{n+1} y_{n}\right\|=\varepsilon \lim _{\mathcal{U}}\left\|T^{n+1} y\right\|>0,
\end{aligned}
$$

since $T$ is power bounded and since $y \notin X_{0}=\operatorname{ker} \mathcal{T}$. We define $\widehat{T}$ on $X_{\mathcal{U}}$ by $\widehat{T}\left(\widehat{\left.x_{n}\right)}=\left(\widehat{T x_{n}}\right)\right.$ and obtain

$$
\operatorname{dist}(\widehat{T} \mathcal{T} y, \mathcal{T}(Y)) \geq \lim _{\mathcal{U}} \operatorname{dist}\left(T^{n+1} y, T^{n}(Y)\right)>0 .
$$

But $\widehat{T} \mathcal{T} y=\left(\widehat{T^{n+1}} y\right)=\mathcal{T} T y \in \mathcal{T}(X)=\mathcal{T}(Y)$, a contradiction.

Let us give some easy examples of power bounded quasi-constricted operators which are not constricted:

Example 1 . Let $X:=C[0,1]$. Define $T: X \rightarrow X$ by $T f(t):=t f(t)$. Then $X_{0}:=\left\{f \in C[0,1]: \lim _{n \rightarrow \infty}\left\|T^{n} f\right\|=0\right\}=\{f \in C[0,1]: f(1)=0\}$ has codimension 1 . So, $T$ is quasi-constricted but not constricted, since it has no nontrivial eigenvectors.

Example 2 ([EW, p. 217]). Let $X=c_{0}$ with sup-norm $\|\cdot\|$. Denote by $e_{k}$ the element of $X$ whose $k$ th coordinate is 1 , and all other coordinates are zero. Fix a real $\alpha \geq 0$ and define the operator $S_{\alpha}: X \rightarrow X$ by

$$
S_{\alpha}\left(e_{k}\right)= \begin{cases}e_{1}+\alpha e_{2} & \text { if } k=1, \\ e_{k+1} & \text { else, }\end{cases}
$$

and let $T_{\alpha}:=\left(I+S_{\alpha}\right) / 2$. It was shown in [EW, Lemma 2.3] that $X_{0}=$ $\left\{x \in X: \lim _{n \rightarrow \infty}\left\|T_{\alpha}^{n} x\right\|=0\right\}=\left\{x \in c_{0}: x_{1}=0\right\}$, and consequently, $\operatorname{codim}\left(X_{0}\right)=1$. So, the operator $T_{\alpha}$ is quasi-constricted. More exactly, $T_{\alpha}$ has an attractor $A_{\alpha}:=\left[-e_{1}, e_{1}\right]+\alpha B_{X}$ such that $\chi\left(A_{\alpha}\right)=\alpha$, and for every $A \in \operatorname{Attr}_{\|\cdot\|}\left(T_{\alpha}\right)$ we have $\chi(A) \geq \alpha$, since the sequence $T_{\alpha}^{n}\left(e_{1}\right)$ is increasing and its supremum in $\ell^{\infty}$ is easily determined as $(1, \alpha, \alpha, \ldots)$. This also implies that when $\alpha>0$ and $\lambda \in \mathbb{C},|\lambda|=1$, then the operator $\lambda T_{\alpha}$ is mean ergodic if and only if $\lambda \notin \sigma_{\pi}\left(T_{\alpha}\right)=\{1\}$. In particular, the operator $T_{\alpha}$ is not constricted for $\alpha>0$.

Take some real $\beta>0$ and consider the equivalent norm $\|\cdot\|_{\beta}$ on $c_{0}$ defined by the formula

$$
\|x\|_{\beta}:=\sup \left\{\left|x_{1}\right|, \beta\left\|x-x_{1} e_{1}\right\|\right\} .
$$

It is easy to see that $\chi_{\|\cdot\|_{\beta}}(A) \geq \alpha \beta$ for every $A \in \operatorname{Attr}_{\|\cdot\|_{\beta}}\left(T_{\alpha}\right)$. In particular, for $\beta=1 / \alpha$ the operator $T_{\alpha}$ has no attractor $A$ satisfying $\chi_{\|\cdot\|_{\beta}}(A)<1$. It should be noted that the operator $T_{\alpha}$ is a contraction with respect to the norm $\|\cdot\|_{\beta}$ whenever $\beta \leq 1 / \alpha$.

EXAMPLE 3 . The same example can also be considered on $\ell^{2}(\mathbb{N})$ where it is an example of a quasi-constricted operator which is neither power 
bounded nor constricted. As we shall see later on, as a consequence of Theorem 7 quasi-constricted power bounded operators on a Hilbert space are constricted.

ExAmPle 4. Here we consider a generalization of Example 2. Let $0 \neq$ $S \in L(X)$ be a power bounded operator such that $\operatorname{codim} \overline{(I-S) X}<\infty$. Without any restriction we may assume that $\|S\|=1$. Take some real $\alpha \in(0,1)$ and consider the operator $T_{\alpha}:=\alpha I+(1-\alpha) S$. The result from Foguel and Weiss [FW, Lemma 2.1] implies

$$
\left\|T_{\alpha}^{n+1}-T_{\alpha}^{n}\right\|=\left\|T_{\alpha}^{n}\left(T_{\alpha}-I\right)\right\|=\left\|(1-\alpha) T_{\alpha}^{n}(I-S)\right\| \rightarrow 0 .
$$

Then $\overline{(I-S) X} \subseteq X_{0}:=\left\{x \in X: \lim _{n \rightarrow \infty}\left\|T_{\alpha}^{n} x\right\|=0\right\}$. So, $\operatorname{codim}\left(X_{0}\right) \leq$ $\operatorname{codim}(I-S) X<\infty$. Consequently, $T_{\alpha}$ is quasi-constricted.

3. On the spectrum of quasi-constricted operators. Let $T$ be a quasi-constricted operator. Let $X=X_{0} \oplus Y$ where $Y$ is an arbitrary finitedimensional complement of $X_{0}$. Let $P$ be the projection from $X$ onto $Y$ with kernel $X_{0}$ and define $Q=I-P, U=Q T Q, V=Q T P$, and $W=P T P$. Since $X_{0}$ is invariant under $T$, we have $P T Q=0$, hence $T=U+V+W$. More precisely, identifying $X$ with $X_{0} \times Y$ we obtain the following matrix representation of $T$ :

$$
T=\left(\begin{array}{cc}
U & V \\
0 & W
\end{array}\right) .
$$

Then $T^{n}$ is represented by the matrix

$$
T^{n}=\left(\begin{array}{cc}
U^{n} & \sum_{k=0}^{n-1} U^{k} V W^{n-1-k} \\
0 & W^{n}
\end{array}\right) .
$$

Let $\varrho(S)$ denote the resolvent set $\mathbb{C} \backslash \sigma(S)$ of the operator $S$. Then an easy calculation shows that for $\lambda \in \varrho(U) \cap \varrho(W)$,

$$
(\lambda-T)^{-1}=\left(\begin{array}{cc}
(\lambda-U)^{-1} & (\lambda-U)^{-1} V(\lambda-W)^{-1} \\
0 & (\lambda-W)^{-1}
\end{array}\right) .
$$

In particular, $\sigma(T) \subset \sigma(U) \cup \sigma(W)$.

The following elementary proposition shows a close connection between the essential spectral radius $r_{\text {ess }}(T)$ and the property of being quasi-constricted.

Proposition 2. Let $T \in L(X)$.

(i) If $r_{\mathrm{ess}}(T)<1$ then $T$ is constricted.

(ii) If $T$ is quasi-constricted then $r_{\mathrm{ess}}(T) \leq 1$.

Proof. (i) Let $r_{\text {ess }}(T)<1$. Let $\beta=\inf \{|\lambda|: \lambda \in \sigma(T),|\lambda| \geq 1\}, \delta=$ $\sup \{|\lambda|: \lambda \in \sigma(T),|\lambda|<1\}$, and 


$$
\gamma= \begin{cases}1 & \text { if } \beta>1, \\ (1+\delta) / 2 & \text { otherwise }\end{cases}
$$

Then the circle $K:=\{z:|z|=\gamma\}$ is in the resolvent set of $T$ and the projection $P=I-(2 \pi i)^{-1} \int_{K}(z-T)^{-1} d z$ maps $X$ onto $P(X)$ which is invariant under $T$ and finite-dimensional because $\sigma(T) \cap\{\lambda:|\lambda|>\delta\}$ consists only of finitely many Riesz points of $\sigma(T)$. Moreover, $\sigma\left(\left.T\right|_{P(X)}\right) \subset\{\lambda:|\lambda| \geq 1\}$. The space $(I-P)(X)=: Z$ is also invariant under $T$ and by construction $r\left(\left.T\right|_{Z}\right)<1$ hence $Z=X_{0}$, and (i) is proved.

(ii) Using the notation of (1) we have $U^{n} \rightarrow 0$ strongly. Therefore, by the Uniform Boundedness Principle, $U$ is power bounded, hence $r(U) \leq 1$. So if $\lambda \in \sigma(T)$ satisfies $|\lambda|>1$ then $\lambda \in \sigma(W)$, and since the subspace $Y$ on which $W$ acts is finite-dimensional, $\lambda$ is an isolated point of $\sigma(T)$. Let $L$ be the spectral projection corresponding to the spectral set $\{\lambda\}$ and set $Z=L(X)$. Then $Z$ is $T$-invariant and $\sigma\left(\left.T\right|_{Z}\right)=\{\lambda\}$. This in turn yields $Z \cap X_{0}=\{0\}$, so $\operatorname{dim}(Z)<\infty$ and hence $\lambda$ is a Riesz point of $T$. Therefore $r_{\text {ess }}(T) \leq 1$.

Now we use this result in order to give a new characterization of the essential spectral radius of an arbitrary bounded operator $T$ on the Banach space $X$. For $T \in L(X)$ we let

$$
\begin{aligned}
& \alpha_{\mathrm{c}}(T):=\inf \left\{\alpha \in \mathbb{R}_{+}: \alpha^{-1} T \text { is constricted }\right\} \\
& \alpha_{\mathrm{q}}(T):=\inf \left\{\alpha \in \mathbb{R}_{+}: \alpha^{-1} T \text { is quasi-constricted }\right\} .
\end{aligned}
$$

Then our proposition yields the following characterization of the essential spectral radius of the operator $T$.

Corollary 3. Let $T \in L(X)$. Then $\alpha_{\mathrm{c}}(T)=\alpha_{\mathrm{q}}(T)=r_{\mathrm{ess}}(T)$.

Proof. Obviously, $\alpha_{\mathrm{q}}(T) \leq \alpha_{\mathrm{c}}(T)$. To finish the proof we show that:

(I) $r_{\mathrm{ess}}(T) \leq \alpha_{\mathrm{q}}(T)$, and

(II) $\alpha_{\mathrm{c}}(T) \leq r_{\mathrm{ess}}(T)$.

(I) Let $\varepsilon>0$. Then $T /\left(\alpha_{\mathrm{q}}(T)+\varepsilon\right)$ is quasi-constricted, hence its essential spectral radius is less than or equal to 1 by the proposition. This in turn yields $r_{\mathrm{ess}}(T) \leq \alpha_{\mathrm{q}}(T)+\varepsilon$ and the assertion follows.

(II) Assume $r_{\text {ess }}(T)<\alpha_{\mathrm{c}}(T)$ and choose $r_{\text {ess }}(T)<\beta<\alpha_{\mathrm{c}}(T)$. Then $r_{\text {ess }}(T / \beta)<1$. By the proposition $T / \beta$ is constricted, a contradiction.

Now we turn to another property of quasi-constricted operators which is a corollary of the next proposition about the spectrum of the operator $W$ (see $(1))$.

Proposition 4. Let $T$ be quasi-constricted and let $X=X_{0} \oplus Y$ be an arbitrary decomposition. For $W$ as in the matrix representation (1) above, $\sigma(W) \subset\{\lambda:|\lambda| \geq 1\}$. 
Proof. Suppose that the assertion fails. Since $Y$ is finite-dimensional there exists an eigenvalue $\lambda$ of $W$ of absolute value strictly less than 1 . Let $e$ be a corresponding eigenvector. Then by (2) we obtain

$$
T^{n}(e)=\lambda^{n} e+\sum_{k=0}^{n-1} \lambda^{n-1-k} U^{k} V e .
$$

Now $V e \in X_{0}$ implies that for every $\varepsilon>0$ there exists $n(\varepsilon)$ such that $\left\|U^{n} V e\right\|<\varepsilon$ for all $n \geq n(\varepsilon)$. Moreover, $U$ is power bounded on $X_{0}$ by the Uniform Boundedness Principle. So set $M=\sup \left\{\left\|U^{n}\right\|: n \in \mathbb{N}\right\}$. Set $N=n(\varepsilon)$ and let $p \geq 1$. Then

$$
\sum_{k=0}^{N+p-1} \lambda^{N+p-1-k} U^{k} V e=\sum_{k=0}^{N-1} \lambda^{N+p-1-k} U^{k} V e+\sum_{k=1}^{p} \lambda^{p-1-k} U^{k} V e
$$

implies

$$
\begin{aligned}
& \left\|\sum_{k=0}^{N+p-1} \lambda^{N+p-1-k} U^{k} V e\right\| \\
& \quad \leq \sum_{k=0}^{N-1}|\lambda|^{N+p-1-k}\left\|U^{k} V e\right\|+\sum_{k=1}^{p}|\lambda|^{p-1-k}\left\|U^{N+k} V e\right\| \\
& \leq|\lambda|^{p} \frac{M\|V e\|}{1-|\lambda|}+\frac{\varepsilon}{|\lambda|(1-|\lambda|)},
\end{aligned}
$$

which yields $\lim _{n \rightarrow \infty} T^{n} e=0$, i.e. $e \in X_{0}$, a contradiction.

COROLlary 5. Let $T$ be a quasi-constricted operator on the Banach space $X$ and let $Z$ be the quotient space $X / X_{0}$. Then the operator $\widetilde{T}$ induced by $T$ on $Z$ satisfies $\sigma(\widetilde{T}) \subset\{\lambda:|\lambda| \geq 1\}$. Moreover, let $X_{0}^{\perp}$ denote the polar of $X_{0}$ in the dual space $X^{*}$. Then $X_{0}^{\perp}$ is $T^{*}$-invariant, isomorphic to the dual space $Z^{*}$ of $Z$ and $\sigma\left(\left.T^{*}\right|_{X_{0}^{\perp}}\right)=\sigma(\widetilde{T})$.

Proof. We use the notation which led to (1). Suppose that the assertion fails. Then there exists an eigenvector $\widetilde{e}$ corresponding to an eigenvalue $\lambda$ of absolute value strictly less than 1 . Since $\widetilde{e} \neq 0$ there exists $e \in Y$ such that $\widetilde{e}=e+X_{0}$. But then $W e=\lambda e$, a contradiction to the proposition above. The remainder is an elementary exercise on polars (see e.g. [Sch2], Chapt. IV, Sect. 1 and 2).

COROLlary 6. Let $T$ be a quasi-constricted operator on the Banach space $X$. Then $\lim _{n \rightarrow \infty} \operatorname{dist}\left(T^{n} x, X_{0}\right)=0$ always implies $x \in X_{0}$.

Proof. The hypothesis implies $\lim _{n \rightarrow \infty} \widetilde{T}^{n} \widetilde{x}=0$ where the $\sim$ denotes the corresponding objects in $Z=X / X_{0}$ (see the foregoing corollary). Since $\sigma(\widetilde{T}) \subset\{\lambda:|\lambda| \geq 1\}$ and $\operatorname{dim}(Z)<\infty$ we obtain $\widetilde{x}=0$. 
Quasi-constricted operators need not be power bounded (see Example 3). However, it is easy to see that for the result of Corollary 6 to hold the operator $T$ need not be quasi-constricted if it is power bounded. For, $X_{0}$ is then obviously closed. Moreover, $\lim _{n \rightarrow \infty} \operatorname{dist}\left(T^{n} x, X_{0}\right)=0$ implies the existence of a sequence $\left(y_{n}\right)_{n}$ in $X_{0}$ satisfying $\lim _{n \rightarrow \infty}\left\|T^{n} x-y_{n}\right\|=0$. The inequality

$$
\left\|T^{k+n} x\right\| \leq\left\|T^{k}\right\| \cdot\left\|T^{n} x-y_{n}\right\|+\left\|T^{k} y_{n}\right\|
$$

yields $\lim \sup _{k}\left\|T^{k} x\right\| \leq M\left\|T^{n} x-y_{n}\right\|$ for fixed $n$ since $y_{n}$ is in $X_{0}$. So $x \in X_{0}$.

On the other hand, let $T$ be an arbitrary bounded linear operator on the Banach space $X$ such that $X_{0}=\left\{x: \lim _{n \rightarrow \infty} T^{n} x=0\right\}$ is closed. Then $\lim _{n \rightarrow \infty} \operatorname{dist}\left(T^{n} x, X_{0}\right)=0$ does not in general imply $\lim _{n \rightarrow \infty} T^{n} x=0$ as the following example shows.

Example 5. Let $H$ be a separable Hilbert space. Define an operator $T \in L(H)$ as follows:

$$
T\left(e_{i, j}\right)= \begin{cases}\alpha_{i} e_{i+1,1}+e_{i, 2}, & j=1, \\ \left(e_{i, j}+e_{i, j+1}\right) / 2, & j>1,\end{cases}
$$

where $\left\{e_{i, j}\right\}_{i, j=1}^{\infty}$ is an orthonormal basis of $H$ and

$$
\alpha_{i}=\left(\sum_{l=1}^{i} l^{-1}\right)^{1 / 3}\left(\sum_{l=1}^{i+1} l^{-1}\right)^{-1 / 3}
$$

for all $i \in \mathbb{N}$. By use of the inequality

$$
2^{-2 l} \sum_{k=0}^{l}\left(\begin{array}{l}
l \\
k
\end{array}\right)^{2} \geq \frac{1}{4 l},
$$

which holds for each $l \in \mathbb{N}$, we obtain

$$
\begin{aligned}
\left\|T^{n+1}\left(e_{i, 1}\right)\right\| & \geq\left\|\left(\prod_{\xi=i}^{n+i-2} \alpha_{\xi}\right) \sum_{l=1}^{n-1} 2^{-l} \sum_{k=0}^{l}\left(\begin{array}{l}
l \\
k
\end{array}\right) e_{n+i-l-1,2+k}\right\| \\
& \geq\left(\sum_{l=1}^{n-1} 2^{-2 l} \sum_{k=0}^{l}\left(\begin{array}{l}
l \\
k
\end{array}\right)^{2}\right)^{1 / 2} \prod_{\xi=i}^{n+i-2} \alpha_{\xi} \geq\left(\frac{1}{4} \sum_{l=1}^{n-1} l^{-1}\right)^{1 / 2} \prod_{\xi=1}^{n-1} \alpha_{\xi} \\
& =\frac{1}{2}\left(\sum_{l=1}^{n-1} l^{-1}\right)^{1 / 2}\left(\sum_{l=1}^{n} l^{-1}\right)^{-1 / 3} \\
& \geq \frac{1}{4}\left(\sum_{l=1}^{n} l^{-1}\right)^{1 / 6}
\end{aligned}
$$

for all $n \geq 2$ and $i \geq 1$. Since $\left\|T^{n} x\right\| \geq\left|x_{i, 1}\right| \cdot\left\|T^{n}\left(e_{i, 1}\right)\right\|$ for $i \in \mathbb{N}$, we have $\lim _{n \rightarrow \infty}\left\|T^{n} x\right\|=\infty$ whenever $x_{i, 1} \neq 0$ for some $i \in \mathbb{N}$. On the other hand, 
it can be shown that

$$
(\forall x \in H)\left[\left[(\forall i \in \mathbb{N})\left[x_{i, 1}=0\right]\right] \Rightarrow \lim _{n \rightarrow \infty}\left\|T^{n} x\right\|=0\right] .
$$

Then $H_{0}:=\left\{x \in H: \lim _{n \rightarrow \infty}\left\|T^{n} x\right\|=0\right\}=\left\{x \in H:(\forall i \in \mathbb{N})\left[x_{i, 1}=0\right]\right\}$ is a closed subspace of $H$. At the same time

$$
\lim _{n \rightarrow \infty} \operatorname{dist}\left(T^{n} x, H_{0}\right)=\lim _{n \rightarrow \infty}\left(\sum_{p=1}^{\infty}\left(\prod_{i=p}^{p+n-1} \alpha_{i}\right)\left|x_{p, 1}\right|^{2}\right)^{1 / 2}=0
$$

for all $x \in H$.

4. When are quasi-constricted operators constricted? Our last result presents a sufficient condition for quasi-constricted operators to be constricted. Let us remark that, for example, a normal quasi-constricted operator on a Hilbert space is always constricted, as is easily seen by use of the spectral measure of the operator.

TheOREM 7. Let $X$ be a Banach space and let $T \in L(X)$ be such that $\bar{\lambda} T$ is mean ergodic for all $\lambda \in \sigma_{\pi}(T)$. Then the following conditions are equivalent:

(i) $T$ is quasi-constricted.

(ii) $T$ is constricted and power bounded.

(iii) $T$ has an attractor $A$ that satisfies $\chi_{\|\cdot\|_{1}}(A)<1$ for some equivalent norm $\|\cdot\|_{1}$ on $X$.

Proof. Let us recall that whenever the bounded operator $T$ is mean ergodic then $\left(T^{n} / n\right)_{n}$ converges strongly to 0 (see $\left.[\mathrm{Kr}], \mathrm{p} .72\right)$. Then by the Uniform Boundedness Principle the sequence is uniformly bounded, which yields in turn $r(T) \leq 1$. In fact, for $r(T)<1$ the assertion of the theorem is trivial. So without loss of generality we assume $r(T)=1$.

(i) $\Rightarrow$ (ii). A unimodular eigenvalue for $T$ is obviously also an eigenvalue for $\widetilde{T}$ on the space $Z$ defined in Corollary 5 . Hence there are only finitely many unimodular eigenvalues $\left\{\lambda_{i}\right\}_{i=1}^{p}$. Let $F_{\lambda_{i}}(T)$ be the subspace of all eigenvectors corresponding to $\lambda_{i}$. Finally, let $E(T)$ be the linear span of all $F_{\lambda_{i}}(T)$. Since $X_{0}$ is closed and has finite codimension and since $F_{\lambda_{i}}(T) \cap$ $X_{0}=\{0\}$, the space $E(T)$ is finite-dimensional, hence closed, and $E(T) \cap$ $X_{0}=\{0\}$. Moreover, each $F_{\lambda_{i}}(T)$ is the space of fixed vectors of the operator $\overline{\lambda_{i}} T$.

Now let $G=X_{0}^{\perp}$. Since $T^{*}$ is weak*-mean ergodic and $G$ is $T^{*}$-invariant and finite-dimensional, $\left.T^{*}\right|_{G}$ is uniformly mean ergodic, which by Corollary 5 implies that $G$ is the span of all eigenspaces corresponding to unimodular eigenvalues of $T^{*}$ (see e.g. exercise 6 on p. 43 of [Sch1]), or in other terms, that $G=E\left(T^{*}\right)$ (see the preceding paragraph). By Sine's famous ergodic 
theorem (see $[\mathrm{Kr}]$, p. 74$), F_{\lambda_{i}}(T)$ separates points in $F_{\lambda_{i}}\left(T^{*}\right)$, hence $E(T)$ separates points in $E\left(T^{*}\right)$, which in turn implies $\operatorname{dim}(E(T)) \geq \operatorname{dim}(G)=$ codim $\left(X_{0}\right)$. So we obtain $X=X_{0} \oplus E(T)$ and (ii) follows.

(ii) $\Rightarrow$ (iii) and (iii) $\Rightarrow$ (i) follow from Theorem 1 .

Corollary 8. Let $T$ be a bounded linear operator on the Banach space $X$ and assume that the semigroup $\left\{T^{n}\right\}_{n=0}^{\infty}$ is weakly almost periodic. The following assertions are equivalent:

(i) $T$ is constricted and power bounded.

(ii) $T$ has a compact attractor.

(iii) $T$ has an attractor $A$ which satisfies $\chi_{\|\cdot\|_{1}}(A)<1$ for some equivalent norm $\|\cdot\|_{1}$.

(iv) $T$ is quasi-constricted.

Proof. One only needs to notice that the semigroup $\left\{(\lambda T)^{n}\right\}_{n=0}^{\infty}$ is weakly almost periodic for all $\lambda \in \mathbb{C},|\lambda|=1$. Then $\lambda T$ is mean ergodic by the Eberlein theorem [Kr, p. 76] for all $\lambda \in \mathbb{C},|\lambda|=1$. The corollary follows immediately from Theorem 7 .

Corollary 9. Let $T$ be a power bounded operator on the Banach space $X$ and assume that $X$ is reflexive. Then $T$ is quasi-constricted if and only if $T$ is constricted.

Proof. The semigroup generated by $T$ is weakly almost periodic since the unit ball of $X$ is weakly compact.

Acknowledgements. We gratefully acknowledge the comments of the referee which enabled us to correct some misprints as well as to make the proof of Theorem 7 more transparent. The proof of the statement in the paragraph preceding Example 5 is also due to him.

The first author is grateful for generous support of the Alexander von Humboldt Foundation during his stay at the University of Tübingen.

\section{References}

[Ba] W. Bartoszek, Asymptotic periodicity of the iterates of positive contractions on Banach lattices, Studia Math. 91 (1988), 179-188.

[EW] E. Yu. Emel'yanov and M. P. H. Wolff, Mean ergodicity on Banach lattices and Banach spaces, Arch. Math. (Basel) 72 (1999), 214-218.

[FW] S. R. Foguel and B. Weiss, On convex power series of a conservative Markov operator, Proc. Amer. Math. Soc. 38 (1973), 325-330.

[Kr] U. Krengel, Ergodic Theorems, de Gruyter, Berlin, 1985.

[LLY] A. Lasota, T. Y. Li and J. A. Yorke, Asymptotic periodicity of the iterates of Markov operators, Trans. Amer. Math. Soc. 286 (1984), 751-764.

[LT] J. Lindenstrauss and L. Tzafriri, Classical Banach Spaces, Vol. I, Ergeb. Math. Grenzgeb. 92, Springer, Berlin, 1977. 
[Sch1] H. H. Schaefer, Banach Lattices and Positive Operators, Grundlehren Math. Wiss. 215, Springer, New York 1974.

[Sch2] H. H. Schaefer (with M. P. H. Wolff), Topological Vector Spaces, 2nd ed., Grad. Texts in Math. 3, Springer, New York, 1999.

[Si] R. Sine, Constricted systems, Rocky Mountain J. Math. 21 (1991), 1373-1383.

Sobolev Institute of Mathematics at Novosibirsk

Acad. Koptyug pr. 4

630090 Novosibirsk, Russia

E-mail: emelanov@math.nsc.ru

Current address:

Mathematisches Institut der

Universität Tübingen

A. D. Morgenstelle 2

D-72076 Tübingen, Germany
Mathematisches Institut der Universität Tübingen

A. D. Morgenstelle 2

D-72076 Tübingen, Germany

E-mail: manfred.wolff@uni-tuebingen.de

Received March 20, 2000

Revised version September 22, 2000 